\title{
Index System for Overall Benefit of Equipment Repair Costs and Its Fuzzy Comprehensive Evaluation
}

\author{
Xu-Sheng Gan ${ }^{1, a}$, Jing-Shun Duanmu, ${ }^{2, b}$, Huan-Bin Wang ${ }^{2, c}$ \\ ${ }^{1}$ XiJing College, Xi'an, Shaanxi, 710123, China \\ ${ }^{2}$ Material Management and Safety Engineering College, Air Force Engineering \\ University, Xi'an, Shaanxi, 710051, China \\ aganxusheng123@163.com, b dmhz521@yahoo.com.cn, cwhb1979@163.com
}

Keywords: Analytic Hierarchy Process; Fuzzy Comprehensive Evaluation; Equipment Repair Costs; Overall Benefit

\begin{abstract}
In order to improve the evaluation for overall benefit of equipment repair costs and provide a good management and decision-making for equipment repair costs, the fuzzy Comprehensive evaluation method is introduced to quantitatively evaluate the overall benefit of equipment repair costs. First the index system for overall benefit evaluation of equipment repair costs is built on the basis of detailed factor analysis, and then Fuzzy theory is used to process the qualitative indices and AHP is used to calculate the weight of each index, finally the three-level fuzzy synthetic evaluation model is established. The example shows that the evaluation method is feasible and effective.
\end{abstract}

\section{Introduction}

The benefit in the use of equipment repair cost is the comparison between the results achieved by repair use spending and the investment amount of used repair cost. Repair activities results may manifest as the physical form and value form. The physical form refers to the quantity and quality of repaired equipment. The value form refers to the improvement of the repair support ability or combat effectiveness, namely military value. It can use the degree that meet the requirement of support capability to measure. The benefit of repair use can is represented by the formula:

$E=V / C$

where $E$ is the using benefit of repair cost, $V$ is the repair activities result, $C$ is repair cost spending.

Under the condition of achieved same results, less the used repair cost, higher the benefit; in the case of certain repair cost expense, more the achievements, higher the benefit. So the using benefit of repair cost has 2 forms; one is to use the same repair cost to obtain greater achievements of repair activities; one is to use the smaller repair cost to achieve the same repair activities results. The using benefit of repair cost is to comprehensive, integrated analyze the gain and loss of the repair expenses in all aspects of the evaluation criteria, and use the comprehensive evaluation method to seek the optimal scheme for repair cost expenditure. Research developed by USA, Japan and Russia and other countries on this field is relatively early, and these countries all have a set of complete benefit evaluation method and program for repair costs. In recent years, our country also pays the attention to the benefit evaluation of equipment repair cost, which starts the implementation phase of the "transformation" strategy.

The overall benefit evaluation of equipment repair costs is a complicated system engineering that includes many uncertain factors. The paper introduces fuzzy theory and uses fuzzy comprehensive evaluation method to analyze the overall benefits of equipment repair costs on the basis of further quantification from the aspects of using benefit of overall repair costs, using benefit of medium repair costs, using benefit of small repair costs, using benefit of equipment costs, using benefit of professional training costs, using benefit of repair management costs. 


\section{Index system for overall benefit evaluation of equipment repair costs}

\section{Setting principle.}

1. Comprehensiveness

From the overall perspective of equipment repair system, every index of the system should be considered from many aspects in order to fully reflect the overall benefit of repair costs in equipment repair system.

2. Rationality

To enhance the working efficiency of equipment repair system, the index system for overall benefit evaluation of repair costs should reasonable set the evaluation parameters for outstanding emphasis, and distinguishing between primariness and secondariness.

\section{Scientificity}

The size of index system for overall benefit evaluation of repair costs should be appropriate, clear define the connotation of various describing parameters, and exclude the compatibility between the indices. To reduce the workload of the evaluation system and ensure the scientificity of evaluation, the indices that have an important impact on the overall benefit of repair costs should be subdivided, other indices should be appropriately divided in the coarse form.

\section{Establishment of index system.}

Considering a good many factors of overall benefit of equipment repair costs, we can establish the evaluation system as shown in Fig. 1.

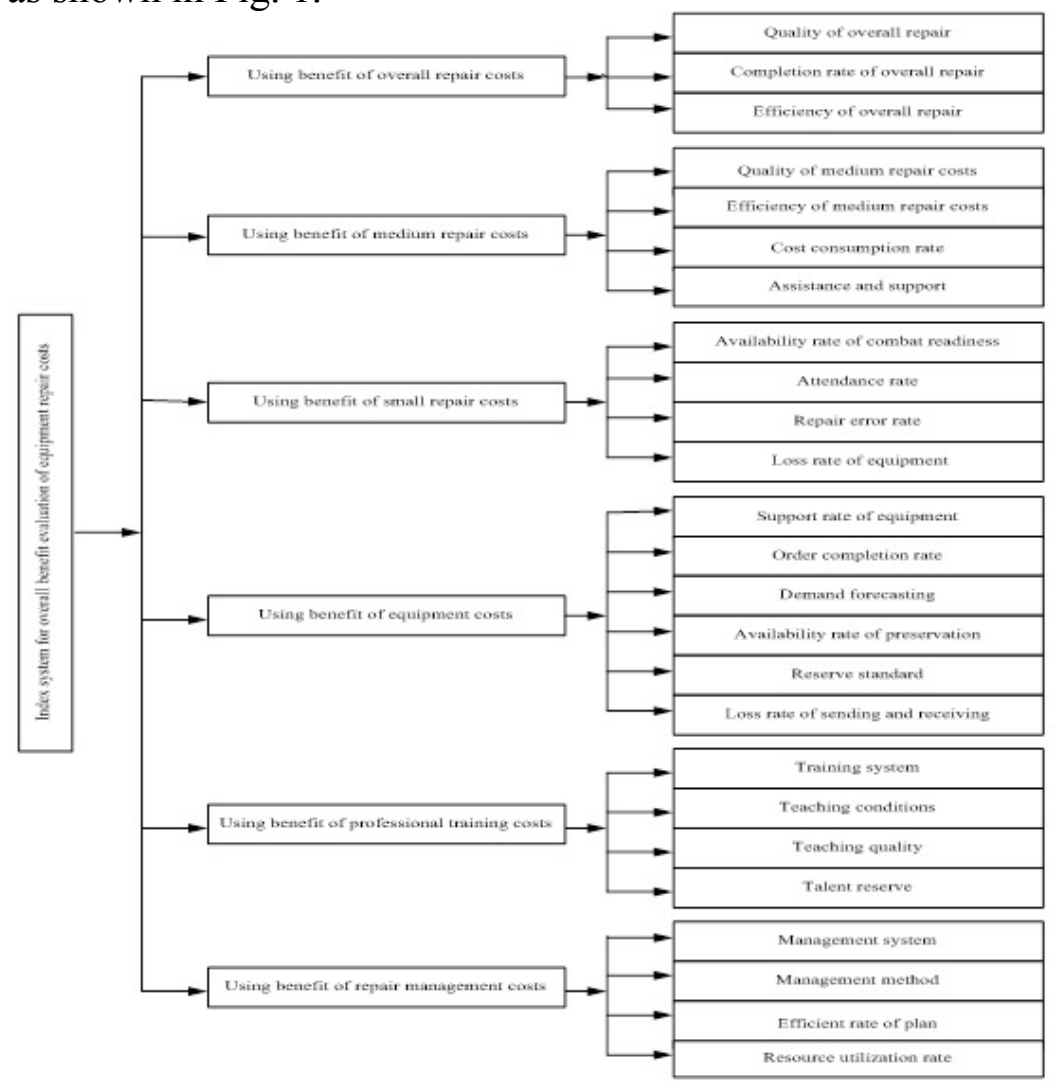

Fig. 1 Index evaluation system for overall benefit of equipment repair costs

\section{Fuzzy comprehensive evaluation method for overall benefit of equipment repair costs}

In index system of overall benefit evaluation of equipment repair costs, there are some quantitative indices such as completion rate of overall repair, availability rate of combat readiness, attendance rate and so on. These indices not only have the different dimension and function relation without comparability, but also their types are also inconsistent. For some indices, the greater, the better. For others indices, the smaller, the better. However, some require the moderation. Thus, these indices need to implement the fuzzification process in advance. To conduct the fuzzy comprehensive 
evaluation, the concept of satisfaction degree is introduced, namely, as far as the idealer system of actual system is considered, their matching degree can be defined as satisfaction degree. For example, for some indices, the greater, the better [1].

$$
G_{m}= \begin{cases}1 & a_{m} \geq S_{m} \\ \frac{a_{m}-s_{m}}{S_{m}-S_{m}} & s_{m}<a_{m}<S_{m} \\ 0 & a_{m} \leq s_{m}\end{cases}
$$

where $p_{m}$ is index value that the bigger, the better. $Q_{m}$ is the ideal maximum; $q_{m}$ is the ideal minimum. Similarly, we can handle the indices that the smaller, the better.

For some indexes that require moderation, we have

$$
G_{f}= \begin{cases}\frac{2\left(a_{f}-s_{f}\right)}{S_{f}-s_{f}} & s_{f} \leq a_{f} \leq \frac{S_{f}-s_{f}}{2} \\ \frac{2\left(S_{f}-a_{f}\right)}{S_{f}-s_{f}} & \frac{S_{f}-s_{f}}{2} \leq a_{f} \leq s_{f} \\ 0 & a_{f}>S_{f}, a_{f}<s_{f}\end{cases}
$$

where $p_{f}$ is the index value that requires moderation. Other indices such as training system, teaching quality, management system and so on, can not be precisely expressed mathematically, but can use the expert scoring method to determine their value. For the evaluation set $U=\left\{U_{1}, U_{2}, U_{3}, U_{4}\right\}$, the expert can mark to a index in terms of the actual situation of the system. then we can suppose $W=\left\{W_{1}, W_{2}, W_{3}, W_{4}\right\}$, so the result of fuzzification process for the indices is $H=W_{g}(U)^{T}, H \in[0,1]$.

In the comprehensive evaluation, the determination of index weight is very important, directly affecting the results of the comprehensive evaluation. In the paper, Analytic Hierarchy Process (AHP) is used to determine the weight that shows the relative importance of each index corresponding to upper level index [2][3]. Because the actual system and ideal system all have some qualitative and fuzzy index requirements, the match requirements can be regarded as a fuzzy set concept, and the so-called satisfaction degree can be regarded as a fuzzy membership degree which need to carry on fuzzy comprehensive evaluation [4][5].

\section{Validation example}

According to above-mentioned fuzzy comprehensive evaluation method and AHP, we can evaluate two schemes of overall benefit of equipment repair costs for a department.

From the results shown in Table 1, It can be seen that: the satisfaction degree of Scheme 1 for overall benefit of equipment repair costs is 0.7947 , obtaining a satisfactory evaluation result. The satisfaction degree of Scheme 2 is 0.6978 . Consequently, for overall benefit of equipment repair costs for a department, Scheme 1 is better than Scheme 2. 
Table 1. Result comparison of fuzzy comprehensive evaluation between Scheme 1 and Scheme 2

\begin{tabular}{|c|c|c|c|c|c|}
\hline \multirow{2}{*}{ Indies of middle level } & \multirow{2}{*}{$\begin{array}{l}\text { Indies weight of } \\
\text { middle level }\end{array}$} & \multirow{2}{*}{ Indies of bottom level } & \multirow{2}{*}{$\begin{array}{l}\text { Indies weight of } \\
\text { bottom level }\end{array}$} & \multicolumn{2}{|c|}{ Fuzzy value } \\
\hline & & & & Scheme 1 & Scheme 2 \\
\hline \multirow{3}{*}{ Using benefit of overall repair costs } & \multirow{3}{*}{0.3060} & Quality of overall repair & 0.4378 & 0.7790 & 0.8245 \\
\hline & & Completion rate of overall repair & 0.3226 & 0.9716 & 0.7826 \\
\hline & & Efficiency of overall repair & 0.2396 & 0.7853 & 0.5844 \\
\hline \multirow{4}{*}{ Using benefit of medium repair costs } & \multirow{4}{*}{0.1343} & Quality of medium repair costs & 0.2448 & 0.8932 & 0.8031 \\
\hline & & Efficiency of medium repair costs & 0.4097 & 0.8451 & 0.7994 \\
\hline & & Cost consumption rate & 0.2032 & 0.6922 & 0.4674 \\
\hline & & Assistance and support & 0.1423 & 0.7744 & 0.4993 \\
\hline \multirow{4}{*}{ Using benefit of small repair costs } & \multirow{4}{*}{0.2695} & Availability rate of combat readiness & 0.3578 & 0.7990 & 0.7082 \\
\hline & & Attendance rate & 0.2942 & 0.8941 & 0.7007 \\
\hline & & Repair error rate & 0.2169 & 0.3744 & 0.4550 \\
\hline & & Loss rate of equipment & 0.1311 & 0.4986 & 0.3445 \\
\hline \multirow{6}{*}{ Using benefit of equipment costs } & \multirow{6}{*}{0.1568} & Support rate of equipment & 0.2732 & 0.8931 & 0.7989 \\
\hline & & Order completion rate & 0.2432 & 0.9247 & 0.9587 \\
\hline & & Demand forecasting & 0.1813 & 0.6443 & 0.8876 \\
\hline & & Availability rate of preservation & 0.1654 & 0.9764 & 0.9428 \\
\hline & & Reserve standard & 0.0988 & 0.7833 & 0.8004 \\
\hline & & Loss rate of sending and receiving & 0.0431 & 0.3249 & 0.5560 \\
\hline \multirow{4}{*}{$\begin{array}{l}\text { Using benefit of professional } \\
\text { training costs }\end{array}$} & \multirow{4}{*}{0.0988} & Training system & 0.2255 & 0.8881 & 0.6940 \\
\hline & & Teaching conditions & 0.2576 & 0.8113 & 0.4472 \\
\hline & & Teaching quality & 0.3748 & 0.8900 & 0.6746 \\
\hline & & Talent reserve & 0.1421 & 0.7666 & 0.6030 \\
\hline \multirow{4}{*}{$\begin{array}{l}\text { Using benefit of repair management } \\
\text { costs }\end{array}$} & \multirow{4}{*}{0.0346} & Management system & 0.3572 & 0.5033 & 0.8047 \\
\hline & & Management method & 0.2028 & 0.6777 & 0.7345 \\
\hline & & Efficient rate of plan & 0.3153 & 0.6790 & 0.6802 \\
\hline & & Resource utilization rate & 0.1247 & 0.7661 & 0.6993 \\
\hline \multicolumn{4}{|c|}{ Fuzzy comprehensive evaluation result for overall benefit of equipment repair costs } & 0.7947 & 0.6978 \\
\hline
\end{tabular}

\section{Conclusions}

The fuzzy comprehensive evaluation method is used to evaluate the overall benefit of equipment repair costs. First, the index system for overall benefit evaluation of equipment repair costs is established by analyzing the influence of many factors, and then the fuzzy treatment of every indies is taken into account. Actual example shows that the evaluation result for overall benefit of equipment repair costs is believable and effective.

\section{References}

[1] D. Y. Chang, L. L. Zhang. The method of fuzzy mathematics in economic and management. Beijing: Beijing Economics Institute Press, (1995)

[2] H. C. Zhao. Analytic hierarchy process. Beijing: Science Press, (1986)

[3] Y. Q. Wei, J. S. Liu, X. Z. Wang. Concept of consistence and weights of the judgement matrix in the uncertain type of AHP. System Engineering and Electronic, 14(4), (1994), 16-22

[4] P. Yang. Fuzzy evaluating model of guarantee capability of equipment and technology of missile army. Modern Defense Technology, 29(4), (2001), 39-41

[5] Y. H. Yue, G. A. Zhou, Y. L. Wang. Fuzzy synthetic evaluation of the surface air defense operational efficiency. System Engineering and Electronic, 23(9), (2001), 36-39 